\title{
The evolution of S0s with UV bright rings A SWIFT-UVOT study
}

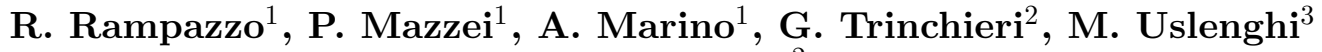 \\ and A. Wolter ${ }^{2}$ \\ ${ }^{1}$ INAF Osservatorio Astronomico di Padova, Vicolo dell'Osservatorio 5, 35122 Padova, Italy \\ ${ }^{2}$ INAF Osservatorio Astronomico di Brera, Via Brera 28, 20121 Milano, Italy \\ ${ }^{3}$ INAF-IASF, Via Bassini 15, 20133 Milano, Italy
}

\begin{abstract}
We report about an ongoing study of the evolution of 24 S0s with UV-bright ring/arm-like structures observed with SWIFT-UVOT and interpreted via SPH simulations with chemo-photometric implementation.
\end{abstract}

Keywords. galaxies: elliptical and lenticular, cD, galaxies: photometry, galaxies: evolution

\section{Introduction}

A significant fraction of S0s is far from being passively evolving. Signatures of activity (e.g. star formation/AGN) maximize their presence in their centre (e.g. Rampazzo et al. 2013). However, spectacular signatures of activity have been extensively mapped by GALEX. FUV bright rings, pseudo-rings and arm-like structures in the disk, shells and tails in the galaxy outskirts are revealed (Thilker et al. 2007; Salim \& Rich 2010; Marino et al. 2011). Sometimes UV-structures are barely visible in the optical bands.

\section{The project and few preliminary results}

We select from the ARRAKIS compilation of galaxies with rings (Comerón et al. 2014) 24 S0s observed with SWIFT-UVOT. We obtained their luminosity profiles and structure and their SED. These information single out, among a grid of SPH simulations with chemo-photometric implementation, the evolution of these S0s providing the history of the development of their ring/arm-like structures (see Mazzei et al. 2014a; Mazzei et al. 2014b). These are found in UV images of S0s with different family classification (see e.g. Laurikainen et al. 2010). UV bright ring/arm-like structures have different shapes, from very diffuse (IC 2006) to filamentary (NGC 1543), to irregular (NGC 1533). SPH simulations suggest that mergers and interactions drive the evolution of ring/arm-like structures which mark transient phases during the galaxy assembly history.

\section{References}

Comerón, S., Salo, H., Laurikainen, E., et al. 2014, A\& A, 562, A121

Laurikainen, E., Salo, H., Buta, R., et al. 2010, MNRAS, 405, 1089

Marino, A., Bianchi, L., Rampazzo, R., et al. 2011, ApJ, 736, 154

Mazzei, P., Marino, A., \& Rampazzo, R. 2014a, ApJ, 782, 53

Mazzei, P., Marino, A., Rampazzo, R., et al. 2014b, Adv. Sp. Res., 53, 950

Salim, S. \& Rich, R. M. 2010, ApJ, 714, L290

Rampazzo, R., Panuzzo, P., Vega, O., et al. 2013, MNRAS, 432, 374

Thilker, D. A., Bianchi, L., Meurer, G., et al. 2007, ApJS, 173, 538 